\title{
A new test apparatus to measure the adhesive shear strength of impact ice on titanium $6 \mathrm{Al}-4 \mathrm{~V}$ alloy
}

\author{
ML.A. Perviera,, B. Gurrutxaga Lerma ${ }^{1}$, E. Piles Moncholi ${ }^{a}$, D.W. \\ Hammond $^{\mathrm{a}}$ \\ ${ }^{a}$ Cranfield university, Cranfield, MK43 OAL, UK \\ ${ }^{b}$ Department of Engineering University of Cambridge, Trumpington St, CB2 1PZ, \\ Cambridge, UK
}

\begin{abstract}
We present a new shear test which may be used in an icing environment. Ice is formed on a jig containing the sample material and this is then loaded by a forcing mechanism to effect the adhesive test. It allows impact (atmospheric) ice adhesive shear tests to be undertaken without disturbance or delay, in icing conditions. Finite element analysis is used in order to evaluate the controlling shear stresses in the most highly stressed zone of the ice/substrate interface and some sample experimental data is given for the adhesion of some impact ices to Ti-6Al-4V alloy with different surface finishes. The adhesion forces reported, represent peak values rather than spatially averaged stress values. Therefore values of adhesive shear strength obtained are higher than previous authors (in the range from 2 to $14 \mathrm{MPa}$ instead of 0.05 to $0.5 \mathrm{MPa}$ ).
\end{abstract}

Keywords: atmospheric ice, impact ice, finite element analysis, shear strength, fracture mechanics

\section{Introduction}

Impact ice (also known as atmospheric ice) is the term for ice formed from 3 supercooled water droplets impinging on a solid body. Such droplets can exist 4 extensively in clouds as nuclei which are able to cause the condensation of 5 a droplet, are often not effective as freezing nuclei until the temperature

Email addresses: m.pervier@cranfield.ac.uk (ML.A. Pervier), bg374@cam.ac.uk (B. Gurrutxaga Lerma) 
falls to several tens of degrees below freezing point [1]. The super-cooled water droplets will however freeze readily on ice particles (producing hail for instance), aircraft, ships, power transmission lines, trees, wind turbines and many other natural and man-made objects leading to a range of hazards.

This study has been conducted for the particular case of ice shedding from aeroengine fan blades. In aircraft engines, fans are not actively protected with any anti-icing or deicing system. Hence, when ice builds up on fan blades, it generally sheds due to the centrifugal force acting on it. Ice pieces can damage the nacelle or be injested by the engine and damage components further down stream. Therefore it is of the highest importance to determine the strength of ice adhesion to the blade in order to allow the ice fragment size to be determined. Furthermore, in the view of the potential to reduce cost of testing, manufacturers are trying to model ice shedding from engine parts and need values for the adhesive strength (in shear and tension) together with the cohesive strength of ice.

Adhesive shear strength has been extensively studied during the last century, however only few authors have reported data on adhesive shear strength of impact ice. Impact ice is quite difficult to obtain. It is necessary to have either a natural site, a cold room or icing tunnel where water can be sprayed. The second difficulty is to have a test apparatus able to work in these conditions. The new test apparatus developped at Cranfield university is able to measure the adhesive shear strength of ice attached to a substrate in a running icing tunnel. Given that the thermal expansion coefficient of ice is generally reported to be approximately 50 microstrains per degree at $-10^{\circ} \mathrm{C}$ (six times that of Titanium) and that significant creep is to be expected at these high homologous temperatures, the means to perform a shear test on ice which is still forming is expected to be of value.

\section{Previous studies}

Two types of shear test have been described for impact ice: "static" tests where the ice is pushed, pulled or twisted to separate it from the body it has grown on, and rotational tests where the ice is removed due to the centrifugal force. These different types of test used lead to a wide range of values reported for the adhesive shear strength (figure 1).

Most of the results fall in the range between 50 and $500 \mathrm{kPa}$. All authors presented a range of values due to the fact that ice is a brittle material which means that scatter will be involved in the results and that several tests need 


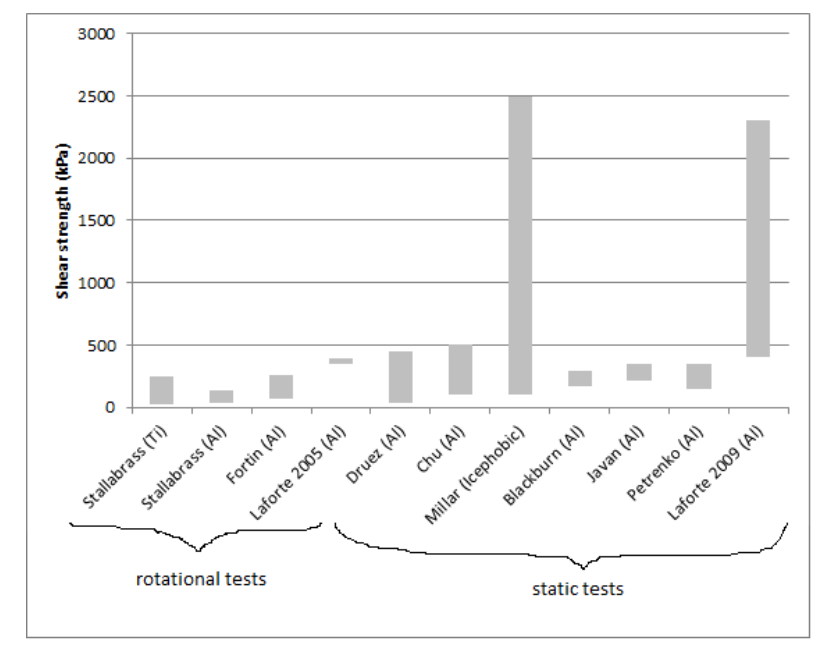

Figure 1: Range of adhesive shear strength values found by the different authors

to be carried out for each icing condition or substrate coating tested. Statistical analysis have been conducted on the experimental results and a mean value and standard deviation have usually been reported. However the scatter does not by itself explain the difference of value reported by the different authors. Three major points could explain the difference in the results: the method of measurements, the different conditions used to form the ice and the properties and the state of the substrate surface.

The first rotational test was carried out by Stallabrass and Price [2]. A cylindrical specimen was mounted on a helicopter rotor blade. Ice was formed by spraying water in a cold room. The blades were rotating at a constant speed of 500 RPM. The centrifugal load was determined using strain gauge measurements. As ice built up, the centrifugal load increased until the adhesive or cohesive strength of ice was reached and ice shed. Five different materials were tested (aluminium, stainless steel, titanium, teflon and viton) through a range of temperature between $-7^{\circ} \mathrm{C}$ and $-18^{\circ} \mathrm{C}$. No special care was taken to clean the blades as it was considered that in application, blades were not cleaned in any way and were contaminated by dust and other sorts of particles. The adhesive shear strength of aluminium and titanium were respectively found to be in the range from 30 to $130 \mathrm{kPa}$ and from 20 to 250 $\mathrm{kPa}$. Whilst this method is realistic for application to spinning components in using centrifugal force to apply the load, it does not force the fracture to follow the interface between the ice and the substrate. Furthermore, it is not 
always possible to see whether the fracture event was confined to the interface (adhesive) or whether the ice broke within itself (cohesive). The authors reported significant cohesive ice fracture with viton and reported that it was difficult to determine the presence or abscence of ice on the metal substrate surface. Therefore, the results do not tell us with certainty what the ice bond strength was.

Fortin and Perron [3] used a similar method but the ice was accreted directly on the blades of a helicopter rotor. The rotating speed was kept constant around 3230 RPM and, as the ice built up, the power needed to rotate the blades increased. An ice shedding event was recorded as a sudden drop in power. The blades were made of aluminium alloy and were resurfaced with scotch brite after each test. Four temperatures spanning the range between $-5^{\circ} \mathrm{C}$ and $-20^{\circ} \mathrm{C}$ have been tested. The adhesive strength is calculated from the balance of the centrifugal, cohesive and adhesive force. The assumption made was that the ice thickness has a linear increase from hub to tip. Values between 70 and $260 \mathrm{kPa}$ were found for the shear strength of ice on Aluminium. Like for the test rig used by Stallabrass and Price, this test rig does not guarantee that an adhesive break can be made. The crack responsible for the fracture will take the easiest path to propagate, either within the ice or at the interface. In case of cohesive failure, the rotational test rigs will then provide lower values for what is taken as the shear strength compared to purely adhesive shear test rigs. In their paper, Stallabrass and Price specified that their results at low temperature were probably underestimated by $50 \%$ due to an overestimation of the area of contact in case of cohesive failure. Laforte and Beisswenger [4] used a slightly different system. Icing was built up at the extremity of beams by spraying water and the beams were then placed in a centrifuge. The speed of the centrifuge was increased from 0 , at a rate of $300 \mathrm{RPM} / \mathrm{s}$, until ice shedding occured. The shedding event was picked up by two piezoelectric cells which can detect vibrations, placed on the side of the centrifuge casing. The shear strength was calculated by dividing the centrifugal force by the iced area. An average value of $350 \mathrm{kPa}$ was obtained for ice on aluminium at a temperature of $-10^{\circ} \mathrm{C}$. Nothing was said about the cleanliness or the roughness of the beams. In this test, only the values when adhesive fracture occurs were kept. The authors specified that cohesive fracture can happen but the tests were discarded.

In general, the rotational tests give lower values than "static" (non-rotating) test rigs. This is probably due to the fact that rotational test rigs are subject to additional forces like vibrations, aerodynamic forces or local heating which 
are not taken into consideration in "static" test rigs. These additional forces are thought to contribute to crack initiation and propagation and therefore results in lower apparent force needed to debond the ice. Whilst rotational tests are probably the best method to test how ice sticks to rotating components, they cannot give a suitable value for pure adhesive shear strength.

Both Druez et al. [5, 6] and Chu and Scavuzzo [7-9] used a test apparatus which pushed the ice accreted around a metalic cylinder. In both cases the ice was formed in an icing tunnel by spraying water on the cold metal surface, then the mechanical test was carried out. In their experiments, Druez et al. used a metal disc to push the ice until it was removed from the surface and the force was recorded by four strain gauges. The shear strength was calculated by dividing the force applied by the contact area between the ice and the substrate. Each adhesion measurement was made at the same temperature as the icing formation but a delay of 20 minutes was observed before any measurement. Substrates were carefully cleaned and dried before ice accretion. Values in the range from 40 to $450 \mathrm{kPa}$ were obtained on aluminium. Substrate of different roughness have been tested and adhesive shear strength has been found to increase with increasing roughness until it reaches a plateau for a roughness of $20 \mu \mathrm{m}$. Chu and Scavuzzo's specimens were made using two concentric cylinders. A window on the outer cylinder allowed ice to stick on the inner cylinder which is made of the metal of interest. The adhesive shear force was measured by pushing the inner cylinder until ice became detached. A load cell was used to record the force and a linear variable displacement transducer to determine the instant of shedding. The test temperature was obtained by heating the interface ice/substrate using a heating element placed at the center of the inner cylinder. The inner cylinder was dipped in acetone and allow to dry. All parts were assembled using tongs to minimise contamination by hand oil. Different material roughness have been tested and this parameter has been found to influence largely the adhesion of ice. Values between 100 and $500 \mathrm{kPa}$ have been obtained depending on the icing conditions. In all these tests, only purely adhesive shear strength values were reported. The authors reported some cohesive failure especially with rime ice but the values were discarded. In these tests the ice is allowed to rest after being built up, so any thermal stresses that could arise as a results of the solidification process will not be involved in the mechanical test. Chu and Scavuzzo even used a different temperature for growing and testing the ice. As the thermal coefficient of expansion of ice is relatively high compared to the thermal coefficient of metal and as the 
ice formation process involves some cooling, a small variation in temperature will induce high thermal stresses which would modify the ice adhesion properties and might lead to a bias of the adhesive shear strength.

Millar [10] has studied the adhesion of ice on a wing. After accretion, a piece of ice is isolated by removing the neighboring ice and then it is pushed using a hydraulic ram device. Values between 100 and $2500 \mathrm{kPa}$ were obtained depending on the material tested (range of icephobic materials like polyurethane, teflon paint or silicone).

The adhesive strength can also be obtained by bending a beam of material which ice is accreted on. Blackburn et al. [11] have argued that, for a specific thickness of ice, when the neutral axis is positioned at the interface ice/substrate, the ice is debonded adhesively and therefore the adhesive shear strength can be obtained. This test was conducted in two steps: the first one where the ice was accreted on aluminium beams in a cold chamber at $-10^{\circ} \mathrm{C}$ and the second one where the iced beams were tested. Several test have been conducted and an average value of $230 \mathrm{kPa}$ has been obtained. No information on LWC, tunnel wind speed or droplet size have been given hence a direct comparison with other values is not possible. Again the ice was fractured in conditions which were not the same as those under which it formed (different static temperature and the ice was allowed to rest after accretion).

Javan-Mashmool et al. [12] also tried to use the bending properties of an aluminium bar to measure the shear strength of ice bonded to it. Prior to the ice accretion, piezoelectric film sensors were attached to the aluminium beams. The iced aluminium beams were clamped onto an electric shaker and the ice adhesion was measured by monitoring bending vibrations. The test temperature was set at $-10^{\circ} \mathrm{C}$ and the wind speed at $3.3 \mathrm{~m} \cdot \mathrm{s}^{-1}$. An average value of $285 \mathrm{kPa}$ was obtained.

Laforte and Laforte [13] reported about other tests to measure the adhesion of ice on an aluminium substrate. They used tests where the ice was only constrained at the interface ice/substrate and the force was applied to the substrate and not to the ice. Due to the applied force, the substrate was strained and the strain propagated into the ice. The force was applied in three different ways: tension, torsion and bending. In all tests, the adhesion of ice was measured in terms of deicing strain directly measured by strain gauges placed on the aluminium bar. Normal stress or shear stress at the instant of shedding can then be calculated from the strain value and the Young's modulus. Only the torsion test gave a value for pure shear strength. 
Average values of 2300, 1000 and $400 \mathrm{kPa}$ were obtained for ice thickness of 2, 5 and $10 \mathrm{~mm}$ respectively. With a thicker sample of ice, the probability of larger defects in the ice increases which results in a lower value of the ice adhesive shear strength. Furthermore, two different materials, aluminium and nylon, have been tested with different surface finishes and results showed an absence of influence of the substrate material but an increase in the shear strength with roughness. Here again time was allowed between ice formation and mechanical test for relaxing the internal stresses.

These tests [5-13] were carried out in two steps: ice was made in one location then moved and was tested in another location. Moving the ice introduce mechanical and thermal shocks that could influence the values obtained [14]. The only static experiment carried out in a running icing tunnel was done by Petrenko [15]. Stainless steel wires of $0.5 \mathrm{~mm}$ in diameter were placed on a surface and, as ice accumulated, the wires were pulled out. The force needed to pull the wires was measured using a force sensor. The time at which the wire was pulled and the tensile force were recorded. The adhesive shear strength of ice was obtained from the measured tensile force and the iced surface of the wires. A curve of adhesive strength variation through time was obtained. For ice made at a temperature of $-10^{\circ} \mathrm{C}$ and a tunnel speed of $20 \mathrm{~m} \cdot \mathrm{s}^{-1}$, values between 150 and $350 \mathrm{kPa}$ were obtained depending on the LWC of the cloud. The thickness of the wires were chosen in such a way that the wires could not stretch as they were pulled out of the ice.

\section{The new ice shear test}

The main objective of this new test is to provide an adhesive shear test which is able to be conducted in a working icing tunnel and provide a shear strength value.

The ice was grown over the face of a plunger and the substrate (figure 2). The substrate has a surface parallel to the direction in which the plunger can be made to move. Once an ice layer of sufficient thickness to provide a satisfactory stress distribution for the test has been accreted, the plunger was pushed with increasing force until the ice becomes detached from the substrate by the shearing action. The pressure needed to move the ice was measured and then converted to a shear strength value through a finite element analysis.

Several test devices could be placed in the tunnel at the same time. Each test device included a substrate, a plunger, a rubber tube and a supporting 


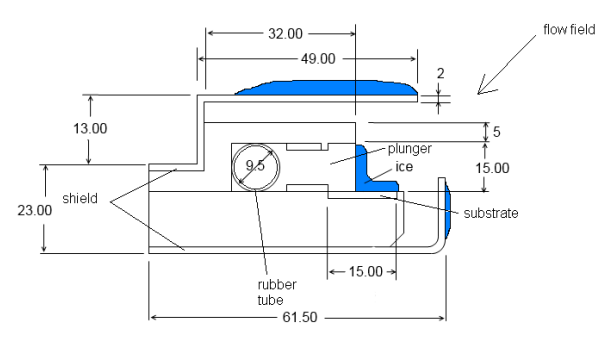

Figure 2: Schematic diagram of the shear test

structure. The substrate can be changed easily so different materials can be tested. Nitrogen gas under pressure passed into the rubber tube which, by inflating, pushed the plunger. The rate at which the gas was allowed into the rubber tube can be varied resulting in controlling the strain rate.

The test rig was placed in the tunnel at an angle of $45^{\circ}$ with respect to the flow stream. In this way both the substrate surface and the plunger wall were uniformly covered by ice. Ice growth on top and bottom parts of the test rig were mainly avoided by the presence of two shields which caught the supercooled water droplets before they impiged on surfaces. This prevented ice from bridging between the moving and the fixed parts. For the same

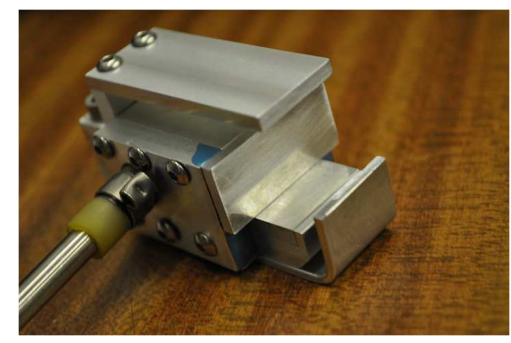

Figure 3: Test rig

reason, the front face of the plunger extended for the full width of the test fixture so that the ice connecting the plunger to the substrate was isolated from other ice on the test fixture (figure 3).

The rubber tube was connected to a source of high pressure nitrogen through 
a pressurization system. The pressurization system consisted of a system of valves allowing several test fixture to be operated independently. A needle valve was employed to select the flow rate of the gas and an electronic valve was used to allow gas to enter the system. A house-made connector consisting of two wires, one positioned on the top of the device and the other between the plunger and the overall structure, was used to determine the instant when the plunger starts to move. The two wires were connected through a little electrical circuit made up of resistors, battery and lights. During the setting of the test, the plunger was pulled back against the structure so the circuit was closed and a current could be measured. The lights were used as a visual indication of the circuit being close or open. When the plunger started to move, the electrical circuit became open, the light went off and a drop in the voltage of the circuit can be observed (figure 4). In most of the cases, the drop of voltage corresponded to a change in the slope of the curve representing the pressure increase through the rubber tube. After the movement

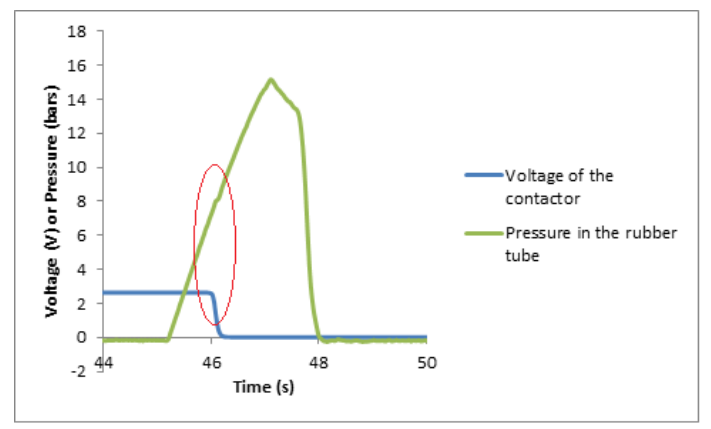

Figure 4: Graph representing the pressure of the gas going through the rubber tube (green curve) and the voltage of the current going through the contactor (blue curve) during a mechanical test

of the plunger (and therefore detachment of ice), the pressure was still able to increase as there was no escape route for the gas. The whole pressurization system had to be purged by the operator manually. The pressure was measured using a pressure transducer and a recording of one value each ms was made by a data acquisition system (DI-718B from DATAQ instruments). The test rigs were placed in the tunnel on two support bars. Attention was made to constrain the rubber tubes well so they can only expand inside the test rig and not on the outside (which can lead to a bursting of the rubber tube). Natural rubber tubing was used because of its exceptional strain capability at the temperature required. 


\section{Test procedure and analysis}

The substrate surfaces were first cleaned with ethanol and dried using a hot air gun. Special care was made to remove any water which might have gone under the plunger. The test jigs were covered and the air supply to the tunnel atomising system was switched on so that any water still in the nozzles can be purged without it landing on the test jigs before the experiment was started. The test jigs were then uncovered, the tunnel working section was closed and the main fan and the cooling system were started. The different parameters (LWC, temperature, tunnel speed) were set and when the tunnel was in stable condition, the water was sprayed.

A thickness of $3 \mathrm{~mm}$ was found to be best to obtain a clean adhesive removal of the ice from the substrate in a single piece. Therefore when such a thickness was reached, which took about 5 minutes, the mechanical test could be started. The tunnel was kept running with the water still being sprayed. Each test device was operated in turn by selecting the individual valve and switching on the electrical valve until the ice sheded. The substrate was visually free of any ice. Therefore the ice fracture mode was assumed to be purely adhesive.

The force applied by the plunger to the ice was calculated from the pressure measured by the pressure transducer taking into account the thickness of the rubber tube walls. The rate at which the test fixture is pressurized was controlled to approximately 10 bars per second for the current investigation. This typically gives fracture in one to two seconds. The strain rate is of the order of $10^{-4} \mathrm{~s}^{-1}$.

A post-processing task consisted of recovering the instant of shedding and noting the value of pressure needed to shed the ice. This latter was called critical pressure measured $\left(P_{c \text { measured }}\right)$. It has to be drawn to the attention of the reader that the value of $P_{c \text { measured }}$ represent the pressure of the gas needed to move the plunger. Hence, to calculate the critical pressure applied on the ice $\left(P_{c}\right)$, the thickness of the rubber tube and the force required to push the plunger when no ice is present need to be taken into consideration. The former point is a coefficient obtained from the geometry of the rubber tube and directly applied to the measured critical pressure value. The latest required an experimental test in a dry icing tunnel at a temperature of $-10^{\circ} \mathrm{C}$. The pressure required to move the plunger was measured at 1.08 bars $\left(P_{\text {cmeasured no ice }}\right)$. This value was used as an offset of the critical pressure 
measured during the test (equation 1).

$$
P_{c}=P_{c \text { measured }} \times \frac{d_{0}-2 e}{d_{0}}-P_{c \text { measured no ice }}
$$

where $d_{0}$ is the diameter and $e$ the thickness of the rubber tube.

As ice is usually considered as a brittle material, the experimental results will include some scatter, even when a lot of care is taken to reproduce the same conditions exactly. To deal with this, several values of critical pressure were obtained for each condition. It has been proved previously that the strength of brittle materials follows a Weibull distribution [16], hence, a statistical analysis was run. The software Statistica ${ }^{1}$ was used and, for each conditions, the software determined the Weibull distribution that best fitted the data (two parameters Weibull distribution). These parameters consist on the shape parameter (or Weibull modulus) which give an indication of the distribution of the flaws in the material, and on the scale parameter which represents the spread of the distribution. A low value of the shape parameter means that the flaws are distributed non-uniformely and that the strength will present more scatter, whereas a high value means a higher reliability in the strength value. The Weibull modulus ranged mainly between 4 and 8 (as a comparison, Weibull modulus for ceramics are in the range from 5 to 20 and about 100 for steel). In a two parameters Weibull distribution the scale parameter is the value at which $63 \%$ of the specimens would have failed. At the end of the process a mean value and a standard deviation were calculated using equations 2 and 3 where $\lambda$ is the scale parameter, $k$ is the Weibull modulus and $\Gamma$ is the Gamma function.

$$
\begin{aligned}
\bar{m} & =\lambda \times \Gamma(1+1 / k) \\
\sigma & =\sqrt{\lambda^{2}\left[\Gamma(1+2 / k)-(\Gamma(1+1 / k))^{2}\right]}
\end{aligned}
$$

\section{Determination of shear strength}

Two different approaches were used to calculate a shear strength value from the critical pressure:

- A shear strength value which is an average over the whole area of ice in contact with the substrate.

\footnotetext{
${ }^{1}$ Statistica is a statictics and analytics sofware developped by StatSoft, http://www . statsoft.com
} 
- A shear strength value which is a peak value related to the most highly stressed region where the plunger, the substrate and the ice meet.

The first value is useful to compare with values reported by other authors while the second is desirable for general modelling efforts away from experiments.

The average value, $\tau_{a v}$, was calculated from the classic definition of shear stress, $\tau=F / A$, where $\tau$ is the shear stress, $\mathrm{F}$ is the force applied and $\mathrm{A}$ is the area of contact. Here $\mathrm{F}$ is the pressure acting on the plunger surface and is equal to $F=P_{c} \times r_{0} \times w$ where $P_{c}$ is the critical pressure needed to move the plunger and therefore detach the ice, $r_{0}$ is the internal diameter of the rubber tube $(0.9 \mathrm{~cm})$ and $w$ is the width of the jig. The area of contact, A, represent the area of substrate in contact with the ice and is equal to $1 \mathrm{~cm}$ times the width of the jig. Hence the average shear strength was obtained from

$$
\tau_{a v}=0.9 \times P_{c}
$$

The average shear stress does not reflect the true stress state at the ice/substrate interface which is affected by edge effects. In particular, even though the applied pressure is constant, the stress distribution at the interface will be non-uniform; its near field will decay with $r^{-1 / 2}$ away from the edge (where the plunger apply the force on ice) of the interface $[17,18]$. Thus, the average shear strength $\tau_{a v}$ will act as a scalable measure of the applied pressure at which the sliding begins. The adhesive shear strength, on the other hand, may only be determined from further considerations regarding the form of the interfacial stress distribution.

The peak shear strength was obtained from Finite Element Modelling. The commercial software Abaqus $9.2^{2}$ has been used for the determination of a correlation between the critical pressure and the stress intensity at the junction between the ice, the interface and the plunger. The local shear strength was then calculated from the latter using the average grain size as a typical flaw size. The use of a finite element analysis allowed us to get the value of the adhesive shear strength through the fracture toughness at the location the force was applied. Therefore, the value obtained will not be an average value along the susbstrate surface but the exact value of shear stress needed

\footnotetext{
${ }^{2}$ Abaqus is the name of a finite element analysis software developped by Simulia, http: //www. simulia.com
} 
to detach the ice at the point where the fracture initiates.

The finite element model, the geometry of which is shown in figure 5, consisted of a rectangular shaped piece representing the substrate, a "L" shaped piece representing the ice and another piece representing the plunger which have a circular wall at the location where the pressure was applied. The

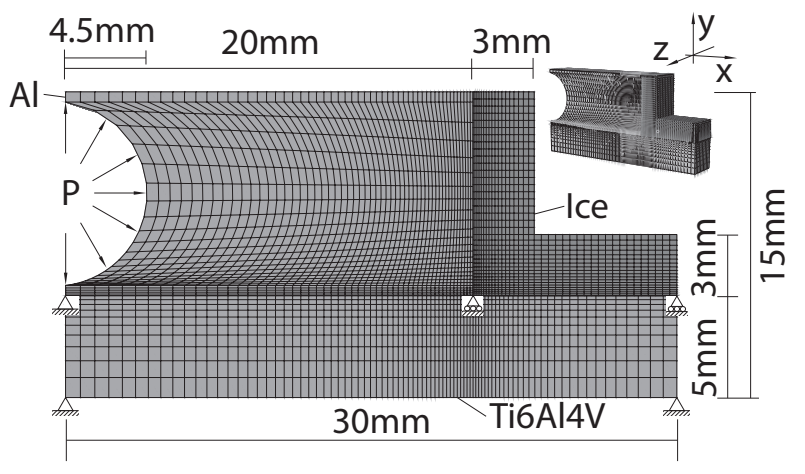

Figure 5: Schematic of the Finite Element Model

plunger and the ice were tied together using a tied constraint, hence these two parts will stay stuck together throughout the whole simulation. The plunger and the substrate were linked with a surface to surface contact interaction with no friction so the plunger was allowed to slide on the substrate surface. The ice and the substrate were linked by a tied constraint. This simulation was made to calculate the shear strength of ice corresponding to the pressure needed to remove the ice. The ice was not supposed to be removed until this pressure was reached, then the ice can be assumed to be completely tied to the substrate for the whole simulation.

During the mechanical test, the gas pressure inflates the rubber tube which will apply pressure to the plunger. To simplify the model, the rubber tube was not represented as a part and the gas pressure was applied directly on the plunger curved wall with an allowance made for the thickness of the rubber tube. The pressure was assumed to be uniform and had a set magnitude. It was applied with a smooth amplitude step to ensure a quasi-static simulation. Two boundary conditions were set: one to restrict the substrate from any movement (encastre boundary condition on the bottom surface of the substrate) and the other to restrict the plunger movements to only translation in the horizontal direction $\mathrm{x}$.

The mesh has been particularly refined at the corner of the "L" shaped ice 
piece as this is the location of the crack initiation. An 8 nodes linear brick element with reduced integration and hourglass control was used for the mesh of all parts. A more detailled discussion on the mesh dependence can be found in [19].

The finite element analysis was conducted in 3D. This was done to account for potential edge effects around the free surfaces, which may have affected the stress distribution along the ice/substrate interface. By gradually reducing the width of the system along the $\mathrm{z}$ direction, we verified that given high translational symmetry of the system and its loading along this z direction, the system works in plane strain.

Furthermore, the observed edge effects were minimal, so we were able to reduce the width without affecting the distribution of stress in the bodies and along the interfaces.

The substrate material was titanium alloy, Ti6Al4V, with a Young's modulus and density of $113 \mathrm{GPa}$ and $4130 \mathrm{~kg} \cdot \mathrm{m}^{-3}$ respectively. Despite the fact that the Young's modulus of ice could vary from 2.5 to $14 \mathrm{GPa}$ and the ice density from 700 to $914 \mathrm{~kg} \cdot \mathrm{m}^{-3}$ depending on the icing conditions, an hypothesis was made that these two parameters were constant over the range of icing conditions explored in the present investigation. A value of $870 \mathrm{~kg} \cdot \mathrm{m}^{-3}$ was chosen for the ice density which is an average value found through the literature. For the Young's modulus of ice, a value of $13.2 \mathrm{GPa}$ was chosen which correspond to an average of the values measured during previous experiments on a few icing conditions (obtained by measuring the speed of sound through ice [19]). These two values are dependent on the ambient conditions used to build the ice. However, as a first approximation and in lack of values, it was assumed that they would be constant throughout the whole range of conditions tested. The plunger material was Aluminium alloy, with a Young's modulus of $70 \mathrm{GPa}$ and a density of $2700 \mathrm{~kg} \cdot \mathrm{m}^{-3}$.

As the pressure value increases, the shear stress build up. The stress field will be near singular at the edge of interface (as shown by Bogy [20]) and away from it decay with $\approx r^{-1 / 2}$ in the near field. A path reading for out putting local stress components was set at the middle of the ice's interface. The values of the shear stress along the path were taken. A value similar to the stress intensity factor, $K_{I I}^{*}$, associated with the interfacial shear stress distribution was defined as

$$
K_{I I}^{*}=\tau \sqrt{2 \pi r}
$$


where $\tau$ is the shear stress and $r$ is the distance from the edge.

The value of the stress intensity factor $K_{I I}^{*}$ when the applied pressure reaches its critical value $P_{c}$ is a universal measure of the interfacial adhesive strength. Barring natural experimental scattering, the critical stress intensity, $K_{I I c}^{*}$, is a characteric of each material and is independent of geometry or loading. In order to determine the $K_{I I c}^{*}$ value from the FEM analysis, the stress intensity factor was calculated from the stress distribution and plotted against the distance from the edge. The curve obtained was fitted by a polynomial equation. The value for $r=0$, was the critical stress intensity $K_{I I c}^{*}$. A correlation can be obtained for different critical pressure applied (figure 6):

$$
K_{I I c}^{*}=182128 \times P_{c}
$$

where the critical pressure, $P_{c}$, is expressed in $\mathrm{MPa}$ and the critical stress intensity factor, $K_{I I c}^{*}$ in $\mathrm{Pa} \mathrm{m}^{1 / 2}$. As may be seen, a linear fit between $K_{I I c}^{*}$ and $P_{c}$ was obtained which is in agreement with theoretical expressions for the contact stress field between wedges and planar surfaces of dissimilar materials [18]. From the value of the critical stress intensity and taking the grain size

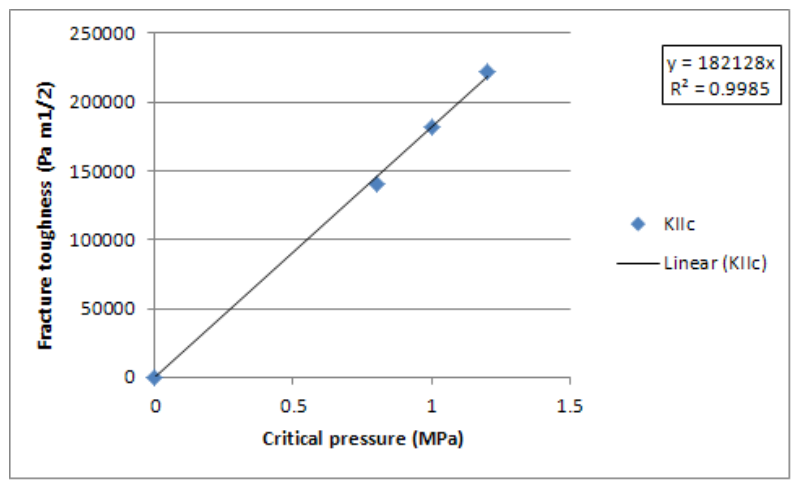

Figure 6: Correlation between critical pressure and fracture toughness

as an indication of material inherent defect size, a shear strength value can be calculated:

$$
\tau=\frac{K_{I I c}^{*}}{\sqrt{\pi a_{g}}}
$$

where $a_{g}$ is the average grain size in $\mathrm{m}$.

The use of $a_{g}$ as the representative lengthscale with which to define the shear strength is justified on the grounds that, give that ice is brittle material, the 
grain size is expected to control the initial crack size and the separation between defects [21-24]. In the following results, the average grain size was obtained from measurement using a nail varnish replica method [25].

\section{Results}

Each result presented was derived statistically from five or more shear tests performed in the same condition. On the graphs, the crosses represent the main value and the error bars represent one standard deviation above and below the main value. Shear strength was obtained using the correlation presented in the previous section and the average grain size measured during microstructure observations. Assumptions have been made that the Young modulus, the poisson ratio and the density of the ice do not vary significantly with tunnel temperature, tunnel wind speed or LWC. The values used are respectively $13.2 \mathrm{GPa}, 0.31$ and $870 \mathrm{~kg} \cdot \mathrm{m}^{-3}$. In all the following, the substrate used was made of titanium alloy Ti-6Al-4V and had a mirror polished finish.

\subsection{Influence of temperature}

The temperature refered to is the total temperature (that is the apparant temperature of the flow once it has been brought to rest) inside the tunnel. It was set prior to the ice accretion process and was kept constant during the whole experiment. The runs made to investigate the influence of temperature have been made using a low and a moderate value of the LWC (respectively 0.4 g. $\mathrm{m}^{-3}$ and $0.7 \mathrm{~g} . \mathrm{m}^{-3}$ ). The tunnel wind speed and the droplet size were kept constant at respectvely $50 \mathrm{~m} \cdot \mathrm{s}^{-1}$ and $20 \mu \mathrm{m}$ for the whole series of experiments.

The shear strength has been found to increase as the temperature decreases in the range of temperature from $-2^{\circ} \mathrm{C}$ to $-12^{\circ} \mathrm{C}$ (figure 7 and 8 ).

The values obtained range between 2.1 and $10.8 \mathrm{MPa}$ which is a lot higher than the values found in the literature. At a temperature of $-10^{\circ} \mathrm{C}$, values less than $500 \mathrm{kPa}$ were usually reported by previous authors. In the present study, the ice was shed from its substrate in exactly the same conditions as during its formation; meaning that no redistribution of thermal stresses has been involved within the ice. Also the shear force reported relates to the peak shear force where fracture initiates, not the mean force/area factor usually used. Shear stress decreases as the distance from the edge increases meaning that an average value would be lower than the value at the edge. 


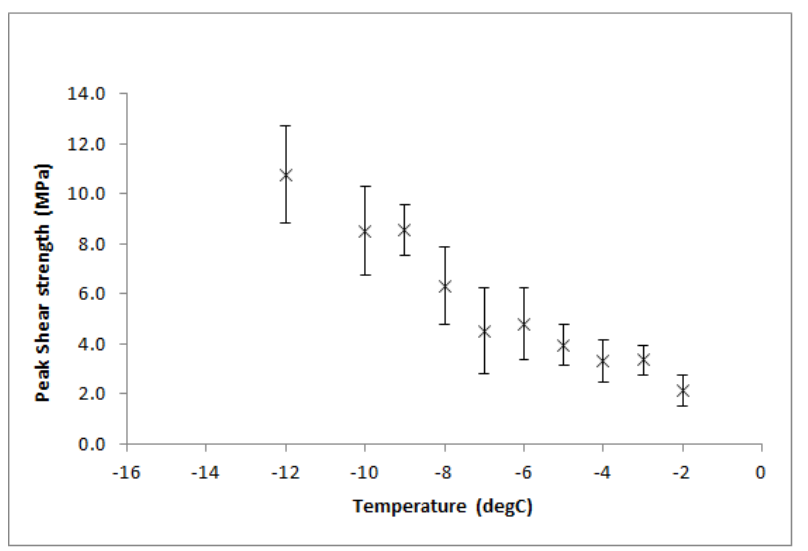

Figure 7: Effect of temperature on the peak shear strength of ice $\left(\mathrm{LWC}=0.4 \mathrm{~g} \cdot \mathrm{m}^{-3}\right)$

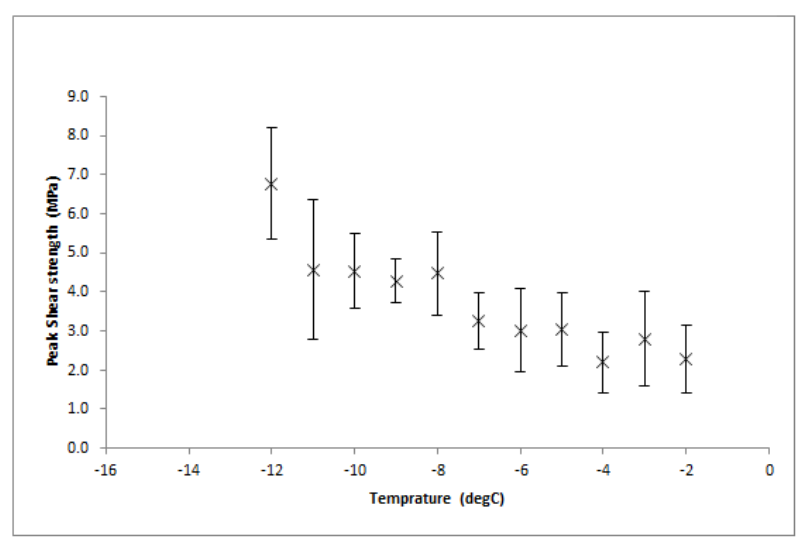

Figure 8: Effect of temperature on the peak shear strength of ice $\left(\mathrm{LWC}=0.7 \mathrm{~g} \cdot \mathrm{m}^{-3}\right)$

By using equation 4, an average shear strength was calculated. The values obtained lie in the range between 0.3 and $1.0 \mathrm{MPa}$ which is closer to the values obtained by previous authors.

The trend of adhesive shear strength to increase with decreasing temperature is relatively comparable with the previous studies. Druez et al. [5, 6], Chu and Scavuzzo [7-9], Stallabrass and Price [2] and Fortin and Perron [3] reported an increase in shear strength as the temperature decreases with either a constant or a maximum value reached at a certain temperature. 


\subsection{Influence of Liquid Water Content (LWC)}

A series of tests has been conducted where the LWC of the cloud has been modified while keeping the tunnel temperature, wind speed and droplet size constant at respectively $-5^{\circ} \mathrm{C}, 50 \mathrm{~m} \cdot \mathrm{s}^{-1}$ and $20 \mu \mathrm{m}$. Five different values of LWC have been tested from 0.4 to $0.8 \mathrm{~g} . \mathrm{m}^{-3}$. Microstructure has been studied at a LWC of $0.4 \mathrm{~g} \cdot \mathrm{m}^{-3}$ and $0.7 \mathrm{~g} \cdot \mathrm{m}^{-3}$ only. Therefore the value of the grains size has been estimated for the other LWC from the known values. Grains have been found to double in size from $225 \mu \mathrm{m}$ at a LWC of $0.4 \mathrm{~g} . \mathrm{m}^{-3}$ to $522 \mu \mathrm{m}$ at a $\mathrm{LWC}$ of $0.7 \mathrm{~g} . \mathrm{m}^{-3}$. In the abscence of microstructure observations at each LWC value and of any trend of behaviour of grains size with LWC, a linear fit was assumed between these two values and extrapolated for the LWC at $0.8 \mathrm{~g} \cdot \mathrm{m}^{-3}$. As this hypothesis could be wrong and therefore mislead the results in terms of peak shear strength, the average shear strength will also be presented and discussed here (figure 9). As

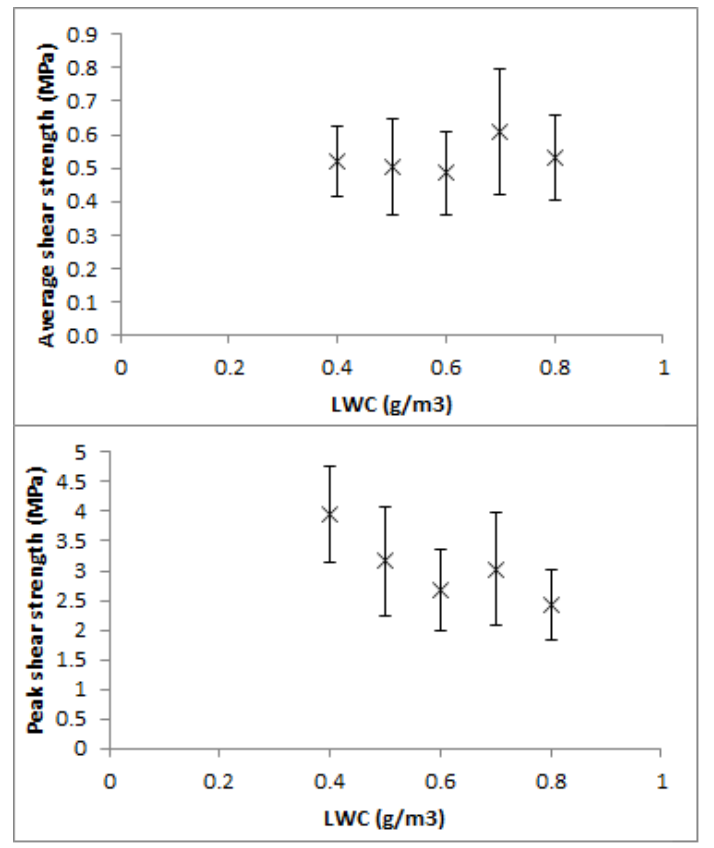

Figure 9: Effect of LWC on the shear strength of ice $\left(\mathrm{T}=-5^{\circ} \mathrm{C}, \mathrm{V}=50 \mathrm{~m} \cdot \mathrm{s}^{-1}, \mathrm{MVD}=20\right.$ $\mu \mathrm{m})$

shown on figure 9, the average shear strength was quasi independent of LWC whereas the peak shear strength was decreasing as the LWC increased. From equation 7 , with a similar value of critical pressure, larger grains size leads 
to lower value of peak shear strength hence the trend observed. Druez et al. [6] conducted experiments with two different LWC and droplet size. He reported that an increase in this combination of parameters resulted in an increase in the adhesive shear strength. The same kind of observation was made by Petrenko [15] who concluded that adhesive shear strength increases with LWC in the range from 0.3 to $2.4 \mathrm{~g} . \mathrm{m}^{-3}$. In these two studies, the wind velocity used was much lower than in the present experiments (between 8 and $20 \mathrm{~m} \cdot \mathrm{s}^{-1}$ for Druez, $20 \mathrm{~m} . \mathrm{s}^{-1}$ for Petrenko and $50 \mathrm{~m} . \mathrm{s}^{-1}$ for this study).

\subsection{Influence of tunnel wind speed}

In the same way as for the previous parameters, the tunnel wind speed has been modified while the temperature, the LWC and the droplet size were kept constant at respectively $-5^{\circ} \mathrm{C}, 0.4 \mathrm{~g} \cdot \mathrm{m}^{-3}$ and $20 \mu \mathrm{m}$. Different values have been tested from 50 to $80 \mathrm{~m} \cdot \mathrm{s}^{-1}$ (figure 10). The average shear strength

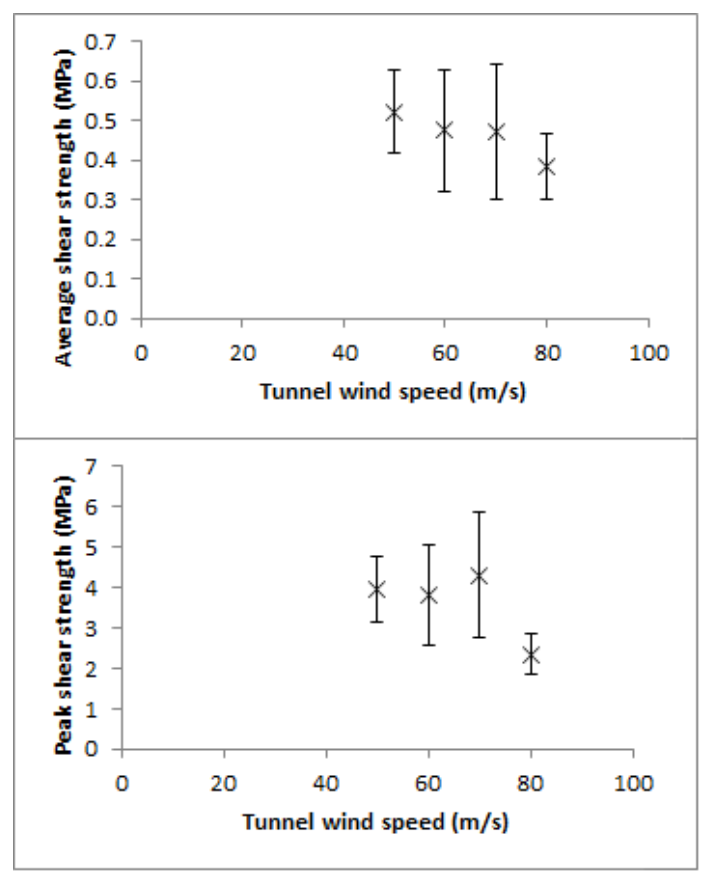

Figure 10: Effect of tunnel wind speed on the shear strength of ice

has been found to decrease from $0.52 \mathrm{MPa}$ to $0.38 \mathrm{MPa}$ as the tunnel wind speed increased from 50 to $80 \mathrm{~m} . \mathrm{s}^{-1}$. The trend of the peak shear strength is less obvious. A maximum seemed to appear at $70 \mathrm{~m} \cdot \mathrm{s}^{-1}$ followed by a 
drop to $80 \mathrm{~m} . \mathrm{s}^{-1}$. More data would be needed to have a better view of the behaviour of peak shear strength with tunnel wind speed.

Druez et al. [5] reported an increase of shear strength with speed from 4 to $16 \mathrm{~m} \cdot \mathrm{s}^{-1}$ which level up until $20 \mathrm{~m} \cdot \mathrm{s}^{-1}$. Chu and Scavuzzo [7] also found a small increase of shear strength with speed between 20 and $90 \mathrm{~m} . \mathrm{s}^{-1}$ but the trend was not clear due to scatter.

\subsection{Influence of surface roughness}

The aforementionned mechanical tests have been carried out on well polished titanium. Some preliminary work of the effect of the substrate surface finish has been made by finishing the titanium surface with coarse grinding paper. This results in the appearance of groves in the horizontal or vertical direction (figure 11). No microstructure observations have been made for
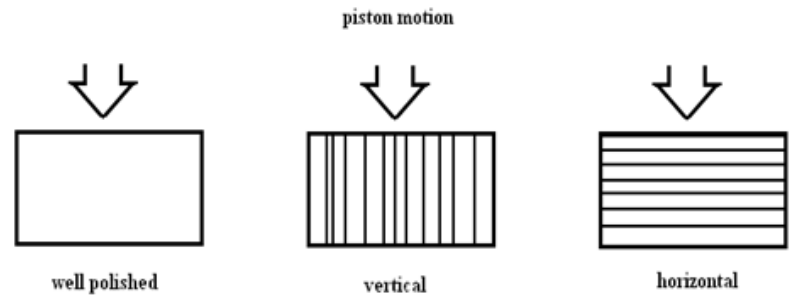

horizontal

Figure 11: Representation of the different roughness on the substrate surface

the ice accreted on these surface so, in order to compare the influence of substrate surface roughness, the average adhesive shear strength will be used in this section.

In general, the adhesive shear strength was seen to increase as the roughness increases and higher values have been found for the horizontal stripes rather than for the vertical stripes. On figure 12, the numbers (500, 800 and 1200) represent the grit of the silicone carbide paper and the letters, $\mathrm{V}$ and $\mathrm{H}$, stands for vertical and horizontal respectively as shown on figure 11 .

The increase in shear strength with the roughness was expected as the ice is assumed to stick more to a rough surface than a smooth surface. In the case of shear especially, ice is thought to slide more easily when accreted on a smoother surface.

The authors who have studied the effect of surface roughness reported an increase in adhesive shear strength as the roughness increases up to a certain 


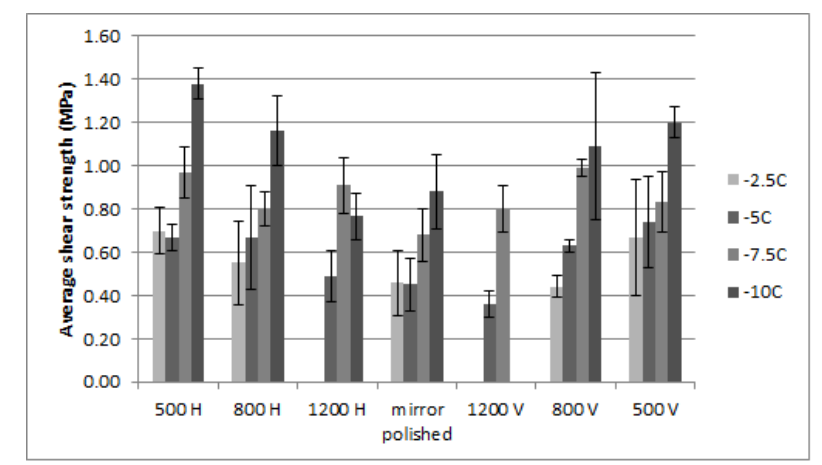

Figure 12: Effect of substrate roughness on the adhesive shear strength of ice

value at which further increase in roughness has no influence on the adhesive shear strength [5, 7-9, 13].

\section{Conclusion}

Whilst many workers have reported ice adhesion strengths to various engineering surfaces, only few workers have done so for impact ice. The work published on impact ice comes from a diversity of test rigs and procedures each producing distinct thermal history and load transfer characteristics. To this range we add a new shear test rig which may be operated in icing conditions. It features a stress concentration to promote adhesive fracture and minimise the influence of ice thickness and any geometrical irregularities. The stress distribution has been analysed in terms of critical stress intensity $K_{I I c}^{*}$ as a function of applied pressure for crack to grow. This has been applied, together with some information on the scale of the microstructure to produce a shear strength.

The test has been used to illlustrate the dependence of shear bond strength of impact ice to a Ti-6Al-4V alloy sheet material on the temperature at which the ice forms and is tested, cloud concentration, wind speed and surface roughness. The trends observed when the ambient temperature was varied, were similar to those reported by other workers but the shear strength values were significantly greater taking this peak stress approach.

\section{Acknowledgements}

This work was supported by Rolls-Royce Plc as part of the Samulet program. 


\section{References}

[1] Hobbs P.V. (1974) Ice Physics. Oxford

[2] Stallabrass J.R., Price R.D. (1962) On the adhesion of ice to various materials. National research laboratories LR-350

[3] Fortin G., Perron J. (2009) Spinning rotor blade tests in icing wind tunnel. AIAA 2009-4260, doi:10.2514/6.2009-4260

[4] Laforte C., Beisswenger A. (2005) Icephobic material centrifuge adhesion test. IWAIS XI

[5] Druez J., Phan C.L., Laforte J.L., Nguyen D.D. (1979) Adhesion of glaze and rime on aluminium electrical conductors. Transaction of the canadian society for mechanical engineering 5:215-220, doi:10.1139/tcsme1978-0033

[6] Druez J., Nguyen D.D., Lavoie Y. (1986) Mechanical properties of atmospheric ice. Cold Regions Science and Technology 13:67-74, doi:10.1016/0165-232X(86)90008-X

[7] Chu M.C., Scavuzzo R.J. (1991) Adhesive shear strength of impact ice. AIAA journal 29:1921-1926

[8] Scavuzzo R.J., Chu M.L., Kellackey C.J. (1996) Structural analysis and properties of impact ices accreted on aircraft structures. NASA CR198473

[9] Scavuzzo R.J., Chu M.L. (1987) Structural properties of impact ices accreted on aircraft structures. NASA CR-179580

[10] Millar D.M. (1970) Investigation of ice accretion characteristics of hydrophobic materials. report no. FAA-DS-70-11

[11] Blackburn C., Laforte C., Laforte J.L. (2000) Apparatus for measuring the adhesion force of a thin ice sheet on a substrate. 9th international workshop of atmospheric icing of structures

[12] Javan-Mashmool M., Volat C., Farzaneh M. (2006) A new method for measuring ice adhesion strength at an ice-substrate interface. Hydrol. Process. 20:645-655, doi:10.1002/hyp.6110 
[13] Laforte C., Laforte J.L. (2009) Tensile, torsional and bending strain at the adhesive rupture of an iced substrat. Proceedings of the ASME 2009 28th international conference on ocean, offshore and arctic engineering pp.79-86, doi:10.1115/OMAE2009-79458

[14] Brouwers E.W., Palacios J.L., Smith E.C., Peterson A.A.(2010) The experimental investigation of a rotor hover icing model with shedding. Annual Forum Proceedings - AHS International, 4, 2619-2635

[15] Petrenko V.F. (2006) In-situ study of physical properties and structure of atmospheric ice. report no.45042-EV

[16] Jayatilaka A. (1979) Fracture energy of engineering brittle materials. Applied science publishers LTD

[17] Anderson T.L. (2017) Fracture mechanics: Fundamentals and applications (4th edition). CRC Press

[18] Johnson K.L. (1985) Contact Mechanics. Cambridge University Press, doi:10.1017/CB09781139171731

[19] Pervier ML.A. (2012) Mechanics of ice detachment applied to turbomachinery. PhD thesis Cranfield University

[20] Bogy (1971) Two edge-bonded elastic wedges of different materials and wedge angles under surface tractions. Journal of Applied Mechanics 38(2):371-386, doi:10.1115/1.3408786

[21] Ashby M.F., Gandhi C. and Taplin D.M.R. (1979) Fracture-mechanism maps and their construction for f.c.c metals and alloys. Acta Metallurgica 27(5):699-729, doi:10.1016/0001-6160(79)90105-6

[22] Cottrell A.H. (1989) Strengths of grain boundaries in pure metals. Materials Science and Technology 5(12):1165-1167, doi:10.1179/mst.1989.5.12.1165

[23] Ashby M.F. and Hallam S.D. (1986) The failure of brittle solids containing small cracks under compressive stress states. Acta Metallurgica 34(3):497-510, doi:10.1016/0001-6160(86)90086-6

[24] Sutton A.P. and Balluffi R.W. (1995) Interfaces in Crystalline Materials. Oxford University Press 
[25] Pervier ML.A., Pervier H., Hammond D.W. (2017) Observation of microstructures of atmospheric ice using a new replica technique. Cold Region Science and Technology 140:54-57, doi:10.1016/j.coldregions.2017.05.002 
2019-02-18

\title{
A new test apparatus to measure the adhesive shear strength of impact ice on titanium 6Al-4V alloy
}

\author{
Pervier, Marie L. A.
}

Elsevier

Pervier MLA, Gurrutxaga Lerma B, Piles Moncholi E, Hammond DW. (2019) A new test apparatus to measure the adhesive shear strength of impact ice on titanium 6AI-4V alloy.

Engineering Fracture Mechanics, Volume 214, June 2019, pp. 212-222

https://doi.org/10.1016/j.engfracmech.2019.01.039

Downloaded from Cranfield Library Services E-Repository 